\title{
Optimal Computation of AC/DC Incremental Transmission Losses Based on Adaptive Slack Bus
}

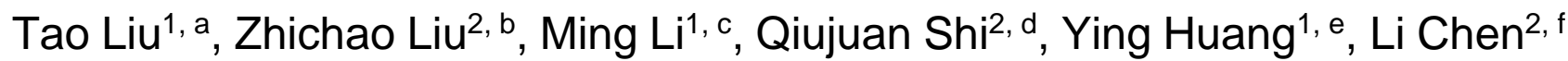 \\ ${ }^{1}$ State Key Laboratory of HVDC, Electric Power Research Institute, China Southern Power Grind, \\ Guangzhou 510080, China;
}

²BEIJING SIFANG AUTOMATION CO., LTD., Beijing, 100085, China.

a569978261@qq.com, b1262579085@qq.com, c1229385928@qq.com, d869221709@qq.com, e1960710665@qq.com, ${ }^{1393447475 @ q q . c o m ~}$

\begin{abstract}
With the development of HVDC transmission technology, AC/DC hybrid system has become an important means of regional power grid interconnection. As a prerequisite of the reliable operation of the system, it is very important to study the power flow calculation and network loss optimization problems of AC/DC hybrid systems for the economic operation of the system. This article first studies the power flow calculation of AC/DC hybrid system, and gives the calculation formula and principle of alternating iterative method. Based on the above conclusions, this article constructs the network loss optimization model of AC/DC hybrid system, and correspondingly puts forward the optimal power flow model and the optimal solution condition that is the law of equal network loss ratio. In this article, due to the redefinition to incremental transmission losses when it is analyzed, a new calculation method is proposed after deriving several commonly used algorithms and applied to the calculation process of network loss optimization. This article also designs and compiles corresponding calculation program, and verifies the correctness of proposed theory and practical method of network loss optimization of AC/DC hybrid system by practical examples.
\end{abstract}

Keywords: AC/DC hybrid system; power flow calculation; slack bus; power network loss optimization; incremental transmission losses.

\section{Introduction}

In the late 1950s, the development of the Yangtze River Three Gorges hydropower station and power transmission problems caused concerns on DC transmission in domestic research. In the 1990s, the successful construction of the Gezhouba-Shanghai South Bridge DC transmission project marked the beginning of the development of DC transmission technology in China. In the whole country, there were more than ten DC transmission projects completed or under construction by the year 2004 [1-5]. The power system transmission mode has been developed towards the direction of ultra-high voltage, high-capacity and long-distance, and researches on power flow calculation and network loss optimization of AC/DC hybrid system has already been put on the agenda. As is known to all that incremental transmission losses is an important indicator to measure whether the system is running optimally, and it has important implications for adjusting network power distribution and reducing loss of the network. This not only can greatly improve the economic benefits, but also makes a contribution to the safe and stable operation of the entire power grid.

Nowadays the method of power flow calculation is very mature in the conventional sense, and at the same time the rapid development of power electronics technology improves the flexibility of HVDC transmission system. The power flow control method of AC/DC hybrid system is more flexible, and VSC-HVDC is a good example which makes remote power transmission more flexible and provides a variety of power flow control means for the power system. In Reference [6], a unified iterative method for AC/DC hybrid system power flow calculation is proposed, aimed at different control modes of VSC, by analyzing DC transmission principle. In Reference [7], the basic principle and construction of VSC-HVDC technology are analyzed in detail and it proposes a power flow calculation method of AC/DC hybrid system based on the power injection model. When it comes to a large-scale AC/DC hybrid system, in Reference [8] it presents a practical model including the MTDC 
system. It contains the inequality constraints that reflect the operation constraints and DC control modes, and uses the interior point method to solve the model. This algorithm is robust and appropriate for large-scale AC/DC power system, because it considers the operation constraints and can converge consistently and steadily in both inner and outer feasible zone of power flow.

In the research of active power optimization of power system, it is very important to study the calculation of incremental transmission losses. It is always necessary to correct the power loss in procedure of dispatching management of power system, and the calculation process of correction factor is the calculation process of incremental transmission losses [9]. There are many methods to calculate the incremental transmission losses. Several classical methods for calculating it are derived in Reference [10] and [11]. In Reference [12], the derived solution of incremental transmission losses, which based on the formula of branch power loss and combined the Jacobi matrix of power flow calculation, can come to the relationship between each branch's power loss and nodal injection power.

In this article, firstly the basic principle and mathematical model of AC/DC hybrid system power flow calculation are discussed. According to the characteristics of AC/DC hybrid system, the mathematical model of network loss optimization is constructed, and the condition for optimal solution of network loss optimization, that is the law of equal network loss ratio, is proposed. Then, several commonly used calculation methods of incremental transmission losses are derived, while their applications in AC/DC hybrid system are analyzed. Based on the current commercial software BPA's principle of algorithm for calculating sensitivity, the algorithm of calculating incremental transmission losses is proposed for power loss changes of the whole transmission channel caused by DC transmission power changes. Finally, according to the law of power loss changes, this article gives some simulation results and proves the correctness of above-mentioned algorithm.

\section{Alternating iterative method applied in power flow calculation}

The power flow calculation equations of AC/DC hybrid system are solved by Newton-Raphson method in the form of polar coordinates. Supposed the number of nodes is n, and the iterative initial values of voltage amplitude and phase angle are the same as those of AC power system. The key issue here is to extend the operational constraints and iterative initial values of variable $\mathrm{X}$. In the iterative calculation process, we can separate the system equations into AC side part and DC side part. In the process of solving the AC side system equations, the DC side system is equivalent to the active load and reactive load of nodes connected with the AC side system. When it comes to solving the DC side system equations, the $\mathrm{AC}$ side system is regarded as constant voltage values of the $\mathrm{AC}$ nodes which belong to the converter.

\subsection{Power flow calculation equations}

For converter $\mathrm{k}$, there are equations:

$$
\begin{aligned}
& V_{d}=k_{T^{*}} V_{t^{*}} \cos \theta_{d}-X_{c^{*}} I_{d^{*}} \\
& V_{d}=k_{\gamma} k_{T^{*}} V_{t^{*}} \cos \varphi \\
& I_{t}=k_{\gamma} k_{T^{*}} I_{d^{*}}
\end{aligned}
$$

In (1) (3), $V_{d}$ and $I_{d}$ are respectively the DC voltage and DC current after commutation of DC system converter; $V_{t}$ is the fundamental component of primary line voltage through the converter transformer on the AC side, $I_{t}$ is the fundamental component of line current, $k_{T}$ is ratio of converter transformer, and $X_{c}$ is equivalent reactance of converter transformer(the converter transformer has been equivalent to ideal transformer); $P_{t s}+j Q_{t s}$ is AC buses power injection; $P_{t d c}+j Q_{t d c}$ is the power of DC system drawn out from AC system; $\varphi$ is power factor angle of the converter, that is the phase angle difference of AC voltage ahead of fundamental voltage. 
According to the state whether a node of AC system is connected with converter transformer, the type of nodes can be set. Set the nodes connected with converter transformers as DC nodes, and set others as AC nodes. Let the number of system nodes be $\mathrm{n}$ and the number of converters in the system be $n_{c}$, then the number of AC nodes will be $n_{a}=n-n_{c}$ because the number of DC nodes is equal to the number of converters. So we can assume that the first $n_{a}$ nodes are AC nodes while the last $n_{c}$ nodes are DC nodes.

The converter transformer and transformed DC system behind on each DC node are equivalent to input and output power $P_{t d c}+j Q_{t d c}$ of the connected DC node, so that the hybrid system can be regarded as an AC network and then the power flow can be solved by conventional power flow calculation procedures and the following calculation equations.

1) Node power equations

As for AC nodes in power system, the power equations of them are:

$$
\left\{\begin{array}{l}
\Delta P_{i}=P_{i s}-V_{i} \sum_{j \in i} V_{j}\left(G_{i j} \cos \theta_{i j}+B_{i j} \sin \theta_{i j}\right)=0 \\
\Delta Q_{i}=Q_{i s}-V_{i} \sum_{j \in i} V_{j}\left(G_{i j} \sin \theta_{i j}-B_{i j} \cos \theta_{i j}\right)=0 \\
i=1,2, \cdots, n_{a}
\end{array}\right.
$$

For the DC nodes, it has been assumed that the converter transformers are connected to ideal filters and converter transformers power loss is negligible. So the power equations can be obtained:

$$
\left\{\begin{array}{l}
\Delta P_{i}=P_{i s}-V_{i} \sum_{j \in i} V_{j}\left(G_{i j} \cos \theta_{i j}+B_{i j} \sin \theta_{i j}\right) \pm V_{d k} I_{d k}=0 \\
\Delta Q_{i}=Q_{i s}-V_{i} \sum_{j \in i} V_{j}\left(G_{i j} \sin \theta_{i j}-B_{i j} \cos \theta_{i j}\right) \pm V_{d k} I_{d k} t g \varphi_{k}=0 \\
i=n_{a}+k \\
k=1,2, \cdots, n_{c}
\end{array}\right.
$$

The parameter $\mathrm{k}$ denotes that the converter transformer numbered $\mathrm{k}$ is connected with the node $\mathrm{i}$, and the positive and negative signs in above equations are respectively corresponding to inverters and rectifiers.

2) Basic equations of converter

According to equation (1) and (2), for converter $\mathrm{k}$, there are following equations:

$$
\begin{gathered}
\Delta d_{1 k}=V_{d k}-k_{T k} V_{n_{a}+k} \cos \theta_{d k}+X_{d k} I_{d k}=0 \\
\Delta d_{2 k}=V_{d k}-k_{\gamma} k_{T k} V_{n_{a}+k} \cos \varphi_{k}=0
\end{gathered}
$$

\subsection{Iterative initial value and operational constraints}

Estimation method is generally used to determine the iterative initial value of expanded variables. For each converter, the initial value of expanded variable can be estimated either by predicting its initial value or by using the given DC power and then by using the fundamental equations of converter. Since the control mode of converter transformer is given, when the variable is estimated, it can be directly given as the value of this variable in the situation of specified control mode. When the type of a bus is PQ bus, the initial value of its voltage can be set as 1.0, and if a bus is PV bus, the value can be set as its voltage setting value; the power factor of converter $\cos \varphi_{d k}$ is set as 0.9 . According to equation (6), if $V_{d k}$ and $k_{T k}$ are both unknown, set the initial value of $k_{T k}$ as 1.0 and then solve the value of $V_{d k}$; if one of the two variables has been known the value, solve the value of the other; if the values of the two variables have been known, solve the value of $\cos \varphi_{d k}$ as the initial value of power factor. According to equation (7), if $I_{d k}$ is unknown, use the predicted DC power to solve its value; if $I_{d k}$ has been known, solve the value of $\cos \theta_{d k}$ as the initial value of $\omega_{k}$. 
Besides, the operational constraints are related to practical situations. The treatment of out-of-limit variables is similar to that of AC system power flow calculation. That is if an expanded variable is out-of-limit, its value is limited to the bounds that it crossed.

\subsection{Power flow calculation equations}

Alternating iterative method is a further simplification of unified iterative method. According to the converter fundamental equations, the influence of AC system on DC system only generates through the primary voltage $V_{t}$ of converter transformer on the DC node. And it shows that if there is a multi-terminal DC system in which the value of $V_{t}$ corresponding to each converter transformer has been known, the DC system equations in power flow calculation equations can be solved separately in addition to the node power equations. And according to node power equations, the influence of DC system on AC system mainly generates through $P_{i d c}+j Q_{i d c}$ that is the power drawn out or injected from AC system of the converter transformer. If $P_{i d c}+j Q_{i d c}$ corresponding to each converter transformer has been known, the changes of parameters in DC system have nothing to do with the power flow calculation of AC system.

Based on the above analysis and conclusions, the iterative process of alternating iterative method is to solve AC system equations and DC system equations separately in equations (4) (7). In the process of solving AC system equations, the DC system is equivalent to the load connected to corresponding DC node whose value is the same as that of the known injected or drawn out active and reactive power. And in the process of solving DC system equations, the AC system is represented as a constant voltage added to the voltage of the converter transformer AC bus. So that in each iteration process, the constant voltage value in the solution of $\mathrm{AC}$ system equations can provide conditions for the solution process of DC system equations, and the solved magnitude of transmission power of DC system equations also provides values of the active and reactive loads on the corresponding node for AC system equations in the next iteration. The above circulation lasts until the equations achieve convergence. In this article, the alternating iterative method uses Newton-Raphson method to solve the equations of AC system and DC system.

\section{Power losses optimization model of AC/DC hybrid system}

\subsection{Mathematical model of power losses optimization}

The losses on system lines are mainly determined by transmission power, line resistance and the operating voltage of each node. Following is the formula of power losses:

$S(u, x)=A(x)+D(u)$

Among the formula:

$$
\begin{aligned}
& x=\left(U_{1}, \cdots, U_{N-r}, \theta_{1}, \cdots, \theta_{N}\right)^{T} \\
& u=\left(P_{d 1}, \cdots, P_{d M}\right) \\
& A(x)=\sum_{i=1}^{N} U_{i} \sum_{j \in i} U_{j} G_{i j} \cos \left(\theta_{i}-\theta_{j}\right) \\
& D(u)=\sum_{k=1}^{M} \frac{P_{d k}^{2}}{U_{d k}^{2}} \cdot R_{d k}
\end{aligned}
$$

In (8), $\mathrm{S}(\mathrm{u}, \mathrm{x})$ is the total power losses of $\mathrm{AC} / \mathrm{DC}$ hybrid system, and $\mathrm{A}(\mathrm{x})$ is $\mathrm{AC}$ system power losses while $\mathrm{D}(\mathrm{x})$ is $\mathrm{DC}$ system power losses; $\mathrm{u}$ is the decision variable, that is the size of DC power adjustment on rectifier station node of DC system; $x$ is the state variable of each node in system including the voltage magnitude $\mathrm{U}$ and phase angle $\theta$.

In the problem of power losses optimization, we mainly change the size of DC power to change system power flow distribution, and then further change the distribution of power losses. When DC power is adjusted, it should be ensured that the real-time data of each section does not exceed the limit and output of power plants and input of each load node do not change. The equations for power losses optimization of AC/DC hybrid system are given as follows: 


$$
\left\{\begin{array}{l}
f\left[x, P_{c}(u), P_{r}(u)\right]=0(\lambda) \\
g(u)<0(\delta)
\end{array}\right.
$$

In (10): $P_{c}(u)$ is rectifier station power, $P_{r}(u)$ is inverter station power; $f($.$) is the power flow$ equation of AC/DC hybrid system; $g(u)$ is constraint equation of rated power on DC side and AC control cross-section of corresponding node; the optimization control variable $u$ is the size of DC power adjustment on rectifier side; $\lambda$ and $\theta$ are lagrange multiplier of the corresponding constraint equations.

Generally speaking, the converter station on DC side is equipped with auto-switching capacitor to enable local reactive power balancing, so the change of reactive power of converter station on DC side has little effect on system power losses. In this article, in order to facilitate the analysis and conclusions, the impact of reactive power changes is considered to be ignored.

In summary, the mathematical model of power losses optimization is that:

In the problem of power losses optimization, we mainly change the size of DC power to change system power flow distribution, and then further change the distribution of power losses. When DC power is adjusted, it should be ensured that the real-time data of each section does not exceed the limit and output of power plants and input of each load node do not change. The equations for power losses optimization of $\mathrm{AC} / \mathrm{DC}$ hybrid system are given as follows:

$$
\left\{\begin{array}{l}
\min S(x, u)=A(x)+D(u) \\
\text { s.t. } f\left[x, P_{c}(u), P_{r}(u)\right]=0(\lambda) \\
g(u)<0(\delta)
\end{array}\right.
$$

\subsection{The optimal condition of power losses optimization}

In this article, the incremental transmission losses of DC system are defined as:

$\nabla_{D}=\frac{\partial D}{\partial u}$

The meaning is that for each additional 1MW transmission power of DC line, the value of increased power losses of the corresponding DC line.

The incremental transmission losses of AC system are defined as:

$$
\nabla_{A}=\frac{\partial A}{\partial x} \frac{\partial x}{\partial P_{c}}-\frac{\partial A}{\partial x} \frac{\partial x}{\partial P_{r}}+\frac{\partial A}{\partial x} \frac{\partial x}{\partial P_{r}} \frac{\partial D}{\partial u}
$$

The meaning is that for each additional 1MW transmission power of DC line, the value of increased power losses of AC system. It can also be called as the incremental transmission losses of AC system about power adjustment amount on DC side.

According to the principle of reduced gradient method, the gradient vector of the power losses objective function to the control variable $\mathrm{u}$ can be obtained as follows:

$$
\frac{\partial S}{\partial u}=\frac{\partial D}{\partial u}-\left(\frac{\partial A}{\partial x} \frac{\partial x}{\partial P_{c}}-\frac{\partial A}{\partial x} \frac{\partial x}{\partial P_{r}}+\frac{\partial A}{\partial x} \frac{\partial x}{\partial P_{r}} \frac{\partial D}{\partial u}\right)
$$

In (14), $\frac{\partial D}{\partial u}$ is the incremental transmission losses of DC system defined hereinbefore; $\frac{\partial A}{\partial x} \frac{\partial x}{\partial P_{c}}-\frac{\partial A}{\partial x} \frac{\partial x}{\partial P_{r}}+\frac{\partial A}{\partial x} \frac{\partial x}{\partial P_{r}} \frac{\partial D}{\partial u}$ is the incremental transmission losses of AC system about power adjustment amount on DC side defined hereinbefore, and in the expression $\frac{\partial A}{\partial x} \frac{\partial x}{\partial P_{c}}$ and $\frac{\partial A}{\partial x} \frac{\partial x}{\partial P_{r}}$ are corresponding to $\mathrm{AC}$ system power losses sensitivity of rectifier station and inverter station respectively.

Therefore, in each step of the iterative process, with the obtained control variables, that is DC transmission power $\mathrm{u}$ of each DC line and the state variable $\mathrm{x}$ which is calculated by substituting itself into power flow calculation, the gradient vector of power losses adjustment can be calculated. After 
the gradient vector $\frac{\partial S}{\partial u}$ has been determined, the adjusted value of control variable can be obtained. And then transmission power of each DC line can be adjusted alone this gradient. The calculation formula of power adjustment amount is that:

$$
P_{d k}^{(n+1)}=P_{d k}^{(n)}+\frac{\partial S}{\partial P_{d k}} \cdot \alpha
$$

In (15), $P_{d k}$ is the result of DC transmission power obtained in the previous step of power flow calculation; $\alpha$ is the optimal step parameter. If during the adjustment process the transmission power value of a certain DC line breaks the cross-section constraint, the transmission power value of the DC line will be fixed at its feasible region boundary $\mathrm{K}$ :

$$
P_{d k}^{(n+1)}=\left\{K \mid P_{d k}^{(n)}+\frac{\partial S}{\partial P_{d k}} \cdot \alpha>K\right\}, \delta \frac{\partial g}{\partial P_{d k}}=\frac{\partial S}{\partial P_{d k}}
$$

When the following conditions of optimal solution are satisfied, the iteration can be stopped, that is, the gradient vector of power adjustment is small enough. Then the system power losses value is minimal with this condition, that is:

$$
\text { If }\left|\frac{\partial S}{\partial u}-\delta^{T} \frac{\partial g}{\partial u}\right|<\varepsilon \text { then stop }
$$

In the process of practical calculation, when properly selecting the position of slack bus, for example, selecting the bus near DC line placement as the slack bus, the AC system power losses sensitivity of inverter station can be very small: $\frac{\partial A}{\partial x} \frac{\partial x}{\partial P_{r}} \approx 0$. In this situation $\nabla_{A}$ can be similarly represented by the AC system power losses sensitivity of rectifier station $\frac{\partial A}{\partial x} \frac{\partial x}{\partial P_{c}}$.

\section{Example and analysis}

In order to verify the power flow calculation of AC/DC hybrid system and the method of power losses optimization of AC/DC hybrid system according to the change of incremental transmission losses proposed in this article, in this chapter, a five-generator 13-bus system is presented as an example. The network nodes wiring diagram of the system is shown in the following figure 1 .

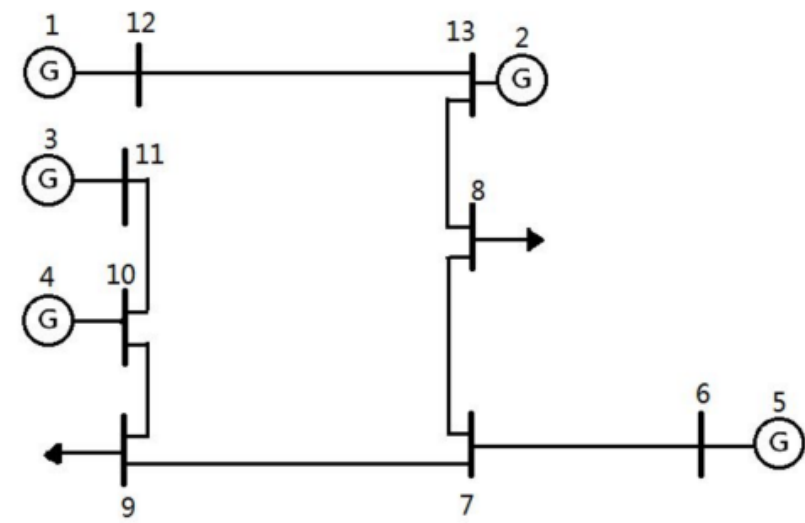

Fig. 1 The network nodes wiring diagram of the system

In the writing diagram, between bus six and bus seven there is a DC line. Select bus three and bus five as slack buses. Write source data file and run the program, and the following results can be obtained: 
Table 1 Power flow calculation solutions at the first time

\begin{tabular}{ccccccc}
\hline $\begin{array}{c}\text { Node } \\
\text { number }\end{array}$ & $\begin{array}{c}\text { Voltage } \\
\text { magnitude }\end{array}$ & $\begin{array}{c}\text { Phase } \\
\text { angle }\end{array}$ & $\begin{array}{c}\text { Output } \\
\text { active } \\
\text { power }\end{array}$ & $\begin{array}{c}\text { Output } \\
\text { reactive } \\
\text { power }\end{array}$ & $\begin{array}{c}\text { Input } \\
\text { active } \\
\text { power }\end{array}$ & $\begin{array}{c}\text { Input } \\
\text { reactive } \\
\text { power }\end{array}$ \\
\hline 1.0000 & 1.0800 & 21.4011 & 5.0000 & 0.8815 & 0 & 0 \\
\hline 2.0000 & 1.0500 & 15.2764 & 5.0000 & 0.1081 & 0 & 0 \\
3.0000 & 1.0800 & 0 & 4.9221 & 1.3918 & 0 & 0 \\
\hline 4.0000 & 1.0500 & -5.1220 & 6.0000 & 1.4515 & 0 & 0 \\
\hline 5.0000 & 1.1000 & 12.0000 & 7.0098 & 1.6126 & 0 & 0 \\
\hline 6.0000 & 1.0808 & 6.3490 & 0.0000 & 0.0000 & 7.0098 & 0.8986 \\
\hline 7.0000 & 1.0438 & 5.2369 & -0.0000 & 0.0000 & -7.0000 & -0.7251 \\
\hline 8.0000 & 1.0485 & 5.7489 & 0.0000 & 0.0000 & 9.6700 & -1.0000 \\
\hline 9.0000 & 1.0127 & -16.3262 & 0.0000 & 0.0000 & 17.6700 & -1.0000 \\
\hline 10.0000 & 1.0313 & -10.4311 & -0.0000 & 0.0000 & 0 & 0 \\
\hline 11.0000 & 1.0612 & -4.1128 & -0.0000 & 0.0000 & 0 & 0 \\
\hline 12.0000 & 1.0692 & 17.2542 & -0.0000 & 0.0000 & 0 & 0 \\
\hline 13.0000 & 1.0513 & 10.9381 & -0.0000 & 0.0000 & 0 & 0 \\
\hline
\end{tabular}

Table 2 Power flow calculation solutions at the first time(continued)

\begin{tabular}{cccccc}
\hline $\begin{array}{c}\text { Starting bus } \\
\text { of a line }\end{array}$ & $\begin{array}{c}\text { Terminal bus } \\
\text { of a line }\end{array}$ & $\begin{array}{c}\text { Input active } \\
\text { power }\end{array}$ & $\begin{array}{c}\text { Input } \\
\text { reactive } \\
\text { power }\end{array}$ & $\begin{array}{c}\text { Output } \\
\text { active power }\end{array}$ & $\begin{array}{c}\text { Output } \\
\text { reactive } \\
\text { power }\end{array}$ \\
\hline 1.0000 & 12.0000 & 5.0000 & 0.8815 & -5.0000 & -0.5124 \\
\hline 2.0000 & 13.0000 & 5.0000 & 0.1081 & -5.0000 & 0.2708 \\
3.0000 & 11.0000 & 4.9221 & 1.3918 & -4.9221 & -1.0172 \\
4.0000 & 10.0000 & 6.0000 & 1.4515 & -6.0000 & -0.8743 \\
\hline 5.0000 & 6.0000 & 7.0098 & 1.6126 & -7.0098 & -0.8986 \\
12.0000 & 13.0000 & 5.0000 & 0.5124 & -4.9447 & -0.0085 \\
13.0000 & 8.0000 & 9.9447 & -0.2623 & -9.8552 & 1.1384 \\
7.0000 & 8.0000 & -0.0925 & -0.1405 & 0.0926 & -0.0692 \\
\hline 7.0000 & 8.0000 & -0.0925 & -0.1405 & 0.0926 & -0.0692 \\
\hline 7.0000 & 9.0000 & 3.5925 & 0.5030 & -3.4584 & 0.6338 \\
\hline 7.0000 & 9.0000 & 3.5925 & 0.5030 & -3.4584 & 0.6338 \\
\hline 9.0000 & 10.0000 & -10.7531 & -0.2676 & 10.8659 & 1.3774 \\
10.0000 & 11.0000 & -4.8659 & -0.5031 & 4.9221 & 1.0172 \\
\hline
\end{tabular}

The value of power losses $\mathrm{S}(\mathrm{u}, \mathrm{x})=5919$

The value of $\mathrm{AC}$ power losses $\mathrm{A}(\mathrm{u}, \mathrm{x})=5821$

The value of DC power losses $\mathrm{D}(\mathrm{u}, \mathrm{x})=0.0098$

The incremental transmission losses of AC system $\nabla_{A}=0.0530$

The incremental transmission losses of DC system $\nabla_{D}=0.0028$

The transmission power of DC lines $P_{c}=7.0098$ 
Table 3 The result of power losses optimization

\begin{tabular}{cccccc}
\hline $\begin{array}{c}\text { Starting bus } \\
\text { of a line }\end{array}$ & $\begin{array}{c}\text { Terminal bus } \\
\text { of a line }\end{array}$ & $\begin{array}{c}\text { Input active } \\
\text { power }\end{array}$ & $\begin{array}{c}\text { Input } \\
\text { reactive } \\
\text { power }\end{array}$ & $\begin{array}{c}\text { Output } \\
\text { active power }\end{array}$ & $\begin{array}{c}\text { Output } \\
\text { reactive } \\
\text { power }\end{array}$ \\
\hline 1.0000 & 12.0000 & 5.0000 & 0.8815 & -5.0000 & -0.5124 \\
\hline 2.0000 & 13.0000 & 5.0000 & 0.1081 & -5.0000 & 0.2708 \\
\hline 3.0000 & 11.0000 & 4.9221 & 1.3918 & -4.9221 & -1.0172 \\
\hline 4.0000 & 10.0000 & 6.0000 & 1.4515 & -6.0000 & -0.8743 \\
\hline 5.0000 & 6.0000 & 7.0098 & 1.6126 & -7.0098 & -0.8986 \\
\hline 12.0000 & 13.0000 & 5.0000 & 0.5124 & -4.9447 & -0.0085 \\
13.0000 & 8.0000 & 9.9447 & -0.2623 & -9.8552 & 1.1384 \\
\hline 7.0000 & 8.0000 & -0.0925 & -0.1405 & 0.0926 & -0.0692 \\
\hline 7.0000 & 8.0000 & -0.0925 & -0.1405 & 0.0926 & -0.0692 \\
\hline 7.0000 & 9.0000 & 3.5925 & 0.5030 & -3.4584 & 0.6338 \\
\hline 7.0000 & 9.0000 & 3.5925 & 0.5030 & -3.4584 & 0.6338 \\
\hline 9.0000 & 10.0000 & -10.7531 & -0.2676 & 10.8659 & 1.3774 \\
\hline 10.0000 & 11.0000 & -4.8659 & -0.5031 & 4.9221 & 1.0172 \\
\hline
\end{tabular}

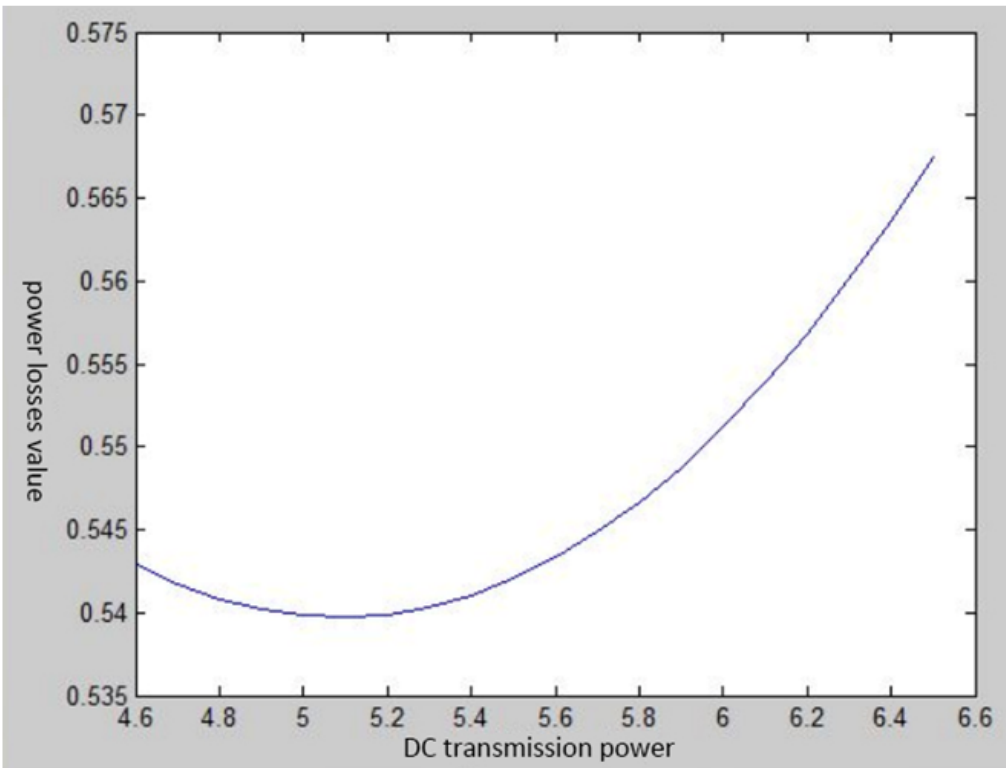

Fig. 2 The relationship schematic diagram of the system total power losses along with the change of DC transmission power

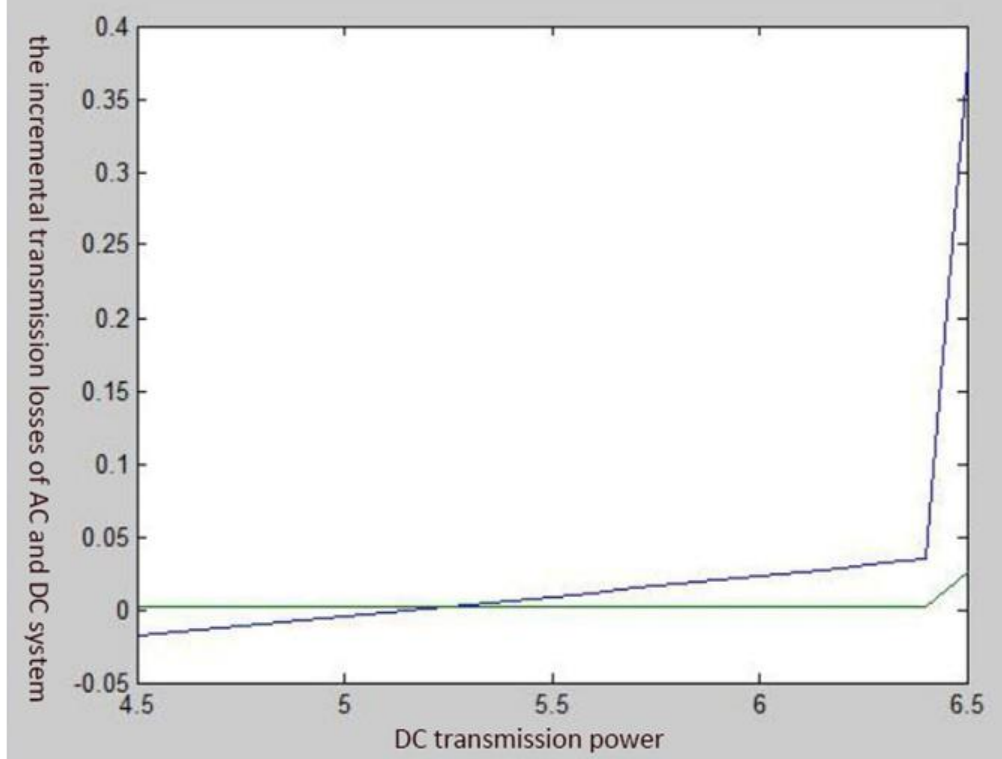

Fig. 3 The schematic diagram of the incremental transmission losses of DC system and AC system along with the change of DC transmission power 
From the table data of table 1,2 and 3, it can be seen that after adjusting the DC power, the value of power losses significantly reduced.

From the simulation results figure 2 and 3, we can find that with the decrease of DC transmission power the system total power losses shows a tendency of first decreasing and then increasing. When the incremental transmission losses of AC system are equal to that of DC system, the power losses value reaches the minimum. The above result verifies the correctness of the proposed algorithm.

\section{Conclusion}

In the process of China's economic rapid development, the power industry occupies the more and more indispensible position in national economy industry. With the continuous development of power industry, the power grid operation requirements are getting higher and higher and the development of HVDC technology is a bigger challenge to modern power system. The expanded scale of AC/DC hybrid system makes power losses reducing have great potential and economic benefits.

In this article, the power flow calculation and power losses optimization of AC/DC hybrid system are analyzed and researched. Power flow calculation is the basic calculation of researching AC/DC hybrid system among that. In AC/DC hybrid system power losses reducing is mainly achieved by changing the DC transmission power value, so that DC active power flow transferred to AC channel and further changes power losses of the whole power system. The adjustment amount of DC transmission power can be obtained by calculating the incremental transmission losses and then minimizes the system power losses.

This article proves the feasibility of proposed theory by writing MATLAB program and running it with practical example. But there are still many problems to be improved, such as the running speed of algorithm for large-scale power grid, and the operation precision of power grid with multiple DC lines and so on. And these problems all need to be further studied in depth.

\section{References}

[1] Wang Xifan, Fang Wanliang, Du Zhengchun. Analysis of Modern Power System. Beijing: Science Press, 2003. p. 166-201.

[2] Zhang Boming, Chen Shousun, Yan Zheng. Advanced Power Network Analysis. 2nd edn. Beijing: Tsinghua University Press, 2007. p. 241-276.

[3] Xu Zheng. Dynamic behavior analysis of AC-DC power system. Beijing: China Machine Press, 2005. p. 52-60.

[4] REN Jianfeng, DING Yawei, FU Lei, et al, J. 'An improved strategy for out-of-step separation based on phase angle principle for $1000 \mathrm{kV}$ ultra-high voltage AC power grids'. Automation of Electric Power Systems. 2011;35(10):104-107.

[5] TANG Guangfu, HE Zhiyuan, PANG Hui, J. 'Research, application and development of VSC-HVDC engineering technology'. Automation of Electrical Power System. 2013; 37(15): 3-14(in Chinese).

[6] TANG Guangfu, LUO Xiang, WEI Xiaoguang, J. 'Multi-terminal HVDC and DC grid technology'. Proceedings of CSEE. 2013; 33(10): 8-17(in Chinese).

[7] ZHANG Yongjun, REN Zhen, LI Bangfeng, J. 'Survey on optimal reactive power dispatch of power system'. Power System Technology. 2005; 29(2):50-56.

[8] Ding Xiaoying, Wang Xifan, J. 'Recent development of optimal power flow in power market'. Automation of Electric Power Systems. 2002; 26(13):1-7(in Chinese). 
[9] Liu Kai, Chen Yiping, Zhang Kun, et al, J. 'Theoretical study of loss minimization for a large AC/DC power system and its implementation in CSG systems'. Proceedings of the CSEE. 2014; 34(1):130-137(in Chinese).

[10] Li Chao'an, J. 'Integrated algorithm of power flow, power losses and incremental transmission losses'. Automation of Electric Power Systems. 1983(5):12-19.

[11] Huang Zhiming. Research and Comparison of Active Power Optimization Algorithm in Power System [D]: [Master Thesis]. Jiangxi: Nanchang University, 2013.

[12] Xu Jianming. Research for active power optimization with classical algorithm and genetic algorithm in power systems [D]: [Master Thesis]. Nanchang: Nanchang University, 2006.

\section{Further Reading}

[13]Zheng Chao, Sheng Canhui, J. 'Uniform iterative power flow algorithm for systems equipped with VSC-HVDCs'. Electric Power. 2007; 40(7):65-69(in Chinese).

[14]Li Gengyin, Zhou Ming, He Jie, Li Guangkai and Liang Haifeng, "Power flow calculation of power systems incorporating VSC-HVDC," Power System Technology, 2004. PowerCon 2004. 2004 International Conference on, 2004, pp. 1562-1566 Vol.2.

[15] QINZhijun, HOUYunhe, WUFuli, J. 'Practical mode lfor large scale AC-DC system power flow calculation'. Proceedings of the CSEE. 2011; 31(10):95-101.

[16]Zhang Liangping, Chang Xianrong, Zheng Huankun, J. 'Calculation Method of Micro-Increasing Rate of Power Loss Based on Branch Power Complex Expression'. Journal of North China Electric Power University. 2011; 38(3):32-35.

[17] M. J. Steinberg and T. H. Smith, "The theory of incremental rates and their practical application to load division-part I," in Transactions of the American Institute of Electrical Engineers, vol. 53, no. 3, pp. 432-445, March 1934.

[18] L. K. Kirchmayer and G. W. Stagg, "Analysis of Total and Incremental Losses in Transmission Systems," in Transactions of the American Institute of Electrical Engineers, vol. 70, no. 2, pp. 1197-1205, July 1951.

[19] T. Senjyu, K. Shimabukuro, K. Uezato and T. Funabashi, "A fast technique for unit commitment problem by extended priority list," Power Engineering Society General Meeting, 2003, IEEE, pp. 882-888.

[20] W. L. Snyder, H. D. Powell and J. C. Rayburn, "Dynamic Programming Approach to Unit Commitment," in IEEE Power Engineering Review, vol. PER-7, no. 5, pp. 41-42, May 1987.

[21]Zhang Xiaoyan. Research on reactive power optimization based on simulated anneal algorithm in distribution network [D]: [ Master Thesis]. Hefei: Hefei University of Technology, 2004

[22]ZHANG Ningyu, GAO Shan, ZHAO Xin, J. 'A fast lagrangian relaxation method for unit commitment'. Power System Protection and Control. 2012; 40(1 9):47-53.

[23] CHEN Ken, LI Xiaoru, XU Min J. 'Comparisons between new method and transposed Jacobian matrix method for calculating incremental transmission losses in active power economic dispatch'. Proceedings of the CSEE. 2000; 20(7):34-36.

[24] Chen Ken, J.M.Kauffmann, Zhang Xiaofeng, Li Jing, Zhong Yun, J. 'Simplifications of Formulas for Calculating Transdon Losses and Incremental Transmission Losses in Rectangular Coodinate Impedance Matrix Method'. Advances in Power System Control, Operation and Management. 2000(1):168-172. 